\title{
THE DIFFEOTOPY GROUP OF THE TWISTED 2-SPHERE BUNDLE OVER THE CIRCLE
}

\author{
M. HO KIM AND FRANK RAYMOND
}

\begin{abstract}
The diffeotopy group of the nontrivial 2-sphere bundle over the circle is shown to be isomorphic to $\mathbb{Z}_{2} \oplus \mathbb{Z}_{2}$. The first generator is induced by a reflection across the base circle, while a second generator comes from rotating the 2-sphere fiber as one travels around the base circle. The technique employed also shows that homotopic diffeomorphisms are diffeotopic.
\end{abstract}

\section{INTRODUCTION}

Gluck proved in [G] that the diffeotopy group $\mathscr{H}$ of $S^{1} \times S^{2}$ is isomorphic to $\mathbb{Z}_{2} \oplus \mathbb{Z}_{2} \oplus \mathbb{Z}_{2}$. In fact, for a large class of 3-dimensional manifolds, Rubinstein, Laudenbach, Waldhausen and others computed the diffeotopy groups. The methods exploited there do not seem to work for the twisted $S^{2}$-bundle over $S^{1}, S^{1} \tilde{\times} S^{2}$. Therefore, we use different methods in conjunction with Gluck's arguments to compute the diffeotopy group $S^{1} \tilde{\times} S^{2}$. In this paper, we shall prove the following:

Theorem. The diffeotopy group $\mathscr{G}$ of the twisted 2-sphere bundle over $S^{1}$, $S^{1} \tilde{\times} S^{2}$, is $\mathbb{Z}_{2} \oplus \mathbb{Z}_{2}$.

Corollary. Each diffeomorphism homotopic to the identity is diffeotopic to the identity.

\section{NOTATION AND PROOF OF THE THEOREM}

We adopt the following notation. Let $I$ be the unit interval $[0,1]$ and $S^{1}$ the unit circle in the plane, i.e., the set of all complex numbers whose absolute value is 1 . We will use $\exp 2 \pi i \theta$ as a point of $S^{1}$, where $\theta$ is a real number and $i$ is $\sqrt{-1}$. Let $S^{2}$ be the unit sphere in the 3-dimensional Euclidean space. We will write $v$ as a point of $S^{2}$. In $S^{1} \tilde{\times} S^{2}, \sim$ means every $(\exp 2 \pi i \theta, v)$ in $S^{1} \times S^{2}$ is identified with $(-\exp 2 \pi i \theta,-v) . D^{2}$ will be the unit disk in the

Received by the editors July 18,1988 .

1980 Mathematics Subject Classification (1985 Revision). Primary 57M99; Secondary 57N37.

Key words and phrases. Diffeotopy, twisted $S^{2}$-bundle over the circle, diffeomorphism, diffeotopy group, $S^{1} \times S^{2}$.

The second author was supported in part by the National Science Foundation. 
plane which is the set of all complex numbers whose absolute value is less than or equal to 1 .

To compute $\mathscr{G}$, we need the following crucial lemma.

(2.1) Lemma. Let $\operatorname{Map}^{1}\left(S^{2}, S^{2}\right)$ be the set of all degree one continuous maps from $S^{2}$ to $S^{2}$. We assume that the topology is induced from the compact open topology.

Define a $\mathbb{Z}_{2}$-action on $\operatorname{Map}^{1}\left(S^{2}, S^{2}\right)$ by

$$
\begin{gathered}
\mathbb{Z}_{2} \times \operatorname{Map}^{1}\left(S^{2}, S^{2}\right) \rightarrow \operatorname{Map}^{1}\left(S^{2}, S^{2}\right) \\
\lambda \rightarrow A \circ \lambda \circ A
\end{gathered}
$$

where $A$ is the antipodal mapping of $S^{2}$. Then the fundamental group of the quotient space is $\mathbb{Z}_{2}$.

Proof. Put $E=\left\{[\alpha] \mid \alpha: I \rightarrow \operatorname{Map}^{1}\left(S^{2}, S^{2}\right), \alpha(0)=\right.$ the identity map $\}$, where $[\alpha]=[\beta]$ means that $\alpha(1)=\beta(1)$ and $\alpha * \bar{\beta}$ is homotopic to a constant path. More precisely, $\alpha * \bar{\beta}$ is the composition of $\alpha$ and $\beta$, i.e.,

$$
\alpha * \bar{\beta}(t)= \begin{cases}\alpha(2 t), & 0 \leq t \leq 1 / 2, \\ \bar{\beta}(2 t-1)=\beta(2-2 t), & 1 / 2 \leq t \leq 1 .\end{cases}
$$

Define $\pi: E \rightarrow \operatorname{Map}^{1}\left(S^{2}, S^{2}\right)$ by $\pi([\alpha])=\alpha(1)$. The space $E$ is simply connected, since $\operatorname{Map}^{1}\left(S^{2}, S^{2}\right)$ is path-connected, and has a universal covering space (cf. [M, p. 394]).

We define two commuting $\mathbb{Z}_{2}$-actions on $E$. The first is given by

$$
(x,[\alpha]) \rightarrow[A \circ \alpha \circ A]
$$

where $x$ denotes the nontrivial element of $\mathbb{Z}_{2}$ in the first action. Next, we are going to use the fact, proved in $[\mathrm{Hu}]$, that $\Pi_{1} \operatorname{Map}^{1}\left(S^{2}, S^{2}\right)=\mathbb{Z}_{2}$. Then, given any path $\alpha$ starting at the identity, we can find a path $\gamma$, also, starting at the identity map with $\alpha(1)=\gamma(1)$ and $\alpha * \bar{\gamma}$ not homotopic to a constant path. We define the second involution

$$
(y,[\alpha]) \rightarrow[\gamma]
$$

where $y$ is the nontrivial element of $\mathbb{Z}_{2}$. This involution describes the generator of the group of covering transformations on $E$. This enables us to conclude that

$$
\Pi_{1}\left(\operatorname{Map}^{1}\left(S^{2}, S^{2}\right) / \mathbb{Z}_{2}\right)=\mathbb{Z}_{2}, \quad \text { i.e. } \quad \Pi_{1}\left(E / \mathbb{Z}_{2} \oplus \mathbb{Z}_{2}\right)=\mathbb{Z}_{2},
$$

because of M. Armstrong's result in [A]:

Let $G$ be a discontinuous group of homeomorphism of a simply connected, locally path connected, Hausdorff space $X$. Then the fundamental group of the quotient $X / G$ is $G / N$ where $N$ is the subgroup of $G$ generated by those elements which have fixed points.

Therefore, we have only to show that there exists no $[\alpha]$ in $E$ such that $x y[\alpha]=[\alpha]$ since $y$ has no fixed point and $x$ has a fixed point. 
Suppose there exists such $[\alpha]$. Then we get

$$
x y[\alpha]=x[\gamma]=[A \circ \gamma \circ A]=[\alpha] .
$$

Since $\alpha(1)=A \circ \gamma(1) \circ A=\gamma(1), \alpha(1)$ is in the set $\mathscr{F}$ of fixed points of the $\mathbb{Z}_{2}$-action on $\operatorname{Map}^{1}\left(S^{2}, S^{2}\right)$. Since $[\gamma]=[A \circ \alpha \circ A],(A \circ \alpha \circ A) * \bar{\gamma}=0$, and $\alpha * \bar{\gamma}$ is not homotopic to a constant path. Since $(A \circ \alpha \circ A) * \bar{\gamma} * \gamma * \bar{\alpha}$ is not homotopic to a constant path, $(A \circ \alpha \circ A) * \bar{\alpha}$ is not homotopic to a constant path. This will lead to a contradiction.

We claim that there is a path $\beta$ in $\operatorname{Map}^{1}\left(S^{2}, S^{2}\right)$ going from identity to $\alpha(1)$ which is fixed under the involution on $\operatorname{Map}^{1}\left(S^{2}, S^{2}\right)$. We use the fact that the set of self-homotopy equivalences of $R P^{2}$ is path-connected (see [GK]). This set corresponds exactly to the set of maps in $\operatorname{Map}^{1}\left(S^{2}, S^{2}\right)$ that are fixed by the given action $\lambda \rightarrow A \circ \lambda \circ A$. So there is a map $\beta: I \rightarrow \operatorname{Map}^{1}\left(S^{2}, S^{2}\right)$ with $\beta(0)=$ identity and $\beta(1)=\alpha(1)$, such that $\beta(t)$ lies in the fixed points set $\mathscr{F}$. Observe that $(A \circ \alpha \circ A) * \bar{\beta} * \beta * \bar{\alpha}$ is not homotopic to a constant path.

Let $\delta=\beta * \bar{\alpha}$. Then $A \circ \delta \circ A=(A \circ \beta \circ A) *(A \circ \bar{\alpha} \circ A)=\beta *(A \circ \bar{\alpha} \circ A)$. Since $\lambda \rightarrow A \circ \lambda \circ A$ is an involution and $\Pi_{1}\left(\operatorname{Map}^{1}\left(S^{2}, S^{2}\right)\right)=\mathbb{Z}_{2}, A \circ \delta \circ A$ is homotopic to $\delta$. Also, each element is its own inverse and $\beta *(A \circ \bar{\alpha} \circ A)$ is homotopic to $(A \circ \alpha \circ A) * \bar{\beta}$. Thus $(A \circ \alpha \circ A) * \bar{\beta} * \beta * \bar{\alpha}$ is homotopic to $\delta * \delta$, which is trivial. This is a contradiction. We have proved the lemma.

(2.2) Corollary. Let $g$ be the self-diffeomorphism of $S^{1} \widetilde{\times} S^{2}$ defined by

$$
[\exp 2 \pi i \theta, v] \rightarrow\left[\exp 2 \pi i \theta,\left(\begin{array}{ccc}
\cos 4 \pi \theta & \sin 4 \pi \theta & 0 \\
-\sin 4 \pi \theta & \cos 4 \pi \theta & 0 \\
0 & 0 & 1
\end{array}\right)(v)\right]
$$

Then $g$ cannot be extended to a map from $\left(D^{2} \times S^{2}\right) / \simeq$ to itself, where $\simeq$ means every point $(r \exp 2 \pi i \theta, v)$ in $D^{2} \times S^{2}$ is identified with $(-r \exp 2 \pi i \theta,-v)$, $0 \leq r \leq 1$.

Proof. Suppose there exists an extension $k$ of $g$. Since $D^{2} \times S^{2}$ is the universal covering space of $\left(D^{2} \times S^{2}\right) / \simeq$, we can lift $k$ to $\tilde{k}$ on $D^{2} \times S^{2}$. Let us examine the value of the second coordinate under the mapping $\tilde{k}$, i.e., consider the following commutative diagram

$$
\begin{array}{ccc}
(r \exp 2 \pi i \theta, v) & (\ldots, K(r \exp 2 \pi i \theta, v)) \\
m & \stackrel{n}{\longrightarrow} & D^{2} \times S^{2} \\
D^{2} \times S^{2} & \downarrow \\
\downarrow & & \\
\left(D^{2} \times S^{2}\right) / \simeq \stackrel{k}{\longrightarrow} & \left(D^{2} \times S^{2}\right) / \simeq
\end{array}
$$

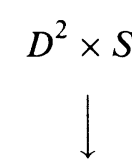


Without loss of generality, since $\operatorname{Map}^{1}\left(S^{2}, S^{2}\right)$ is path-connected, we may assume that $K$ is a map from $D^{2} \times S^{2}$ to $S^{2}$ such that

$$
\begin{gathered}
K(\exp 2 \pi i \theta, v)=\left(\begin{array}{ccc}
\cos 4 \pi \theta & \sin 4 \pi \theta & 0 \\
-\sin 4 \pi \theta & \cos 4 \pi \theta & 0 \\
0 & 0 & 1
\end{array}\right)(v), \\
K(0, v)=\left(\begin{array}{lll}
1 & 0 & 0 \\
0 & 1 & 0 \\
0 & 0 & 1
\end{array}\right)(v)
\end{gathered}
$$

and

$$
-K(-r \exp 2 \pi i \theta,-v)=K(r \exp 2 \pi i \theta, v) .
$$

Define $\widetilde{K}$ from $D^{2}$ to $\operatorname{Map}^{1}\left(S^{2}, S^{2}\right)$ by

$$
r \exp 2 \pi i \theta \rightarrow K(r \exp 2 \pi i \theta, \ldots) \text {. }
$$

From the diagram above, we get $\widetilde{K}(-r \exp 2 \pi i \theta)=A \circ \widetilde{K}(r \exp 2 \pi i \theta) \circ A$. Consider $p \circ \widetilde{K}$. Then $p \circ \widetilde{K}(-r \exp 2 \pi i \theta)=p \circ \widetilde{K}(r \exp 2 \pi i \theta)$ where $p$ is the projection from $\operatorname{Map}^{1}\left(S^{2}, S^{2}\right)$ to $\operatorname{Map}^{1}\left(S^{2}, S^{2}\right) / \mathbb{Z}_{2}$.

Define $\tilde{\tilde{K}}$ from $D^{2}$ to $\operatorname{Map}^{1}\left(S^{2}, S^{2}\right) / \mathbb{Z}_{2}$ by

$$
\tilde{\tilde{K}}(r \exp 2 \pi i \theta)=p \circ \widetilde{K}(r \exp \pi i \theta) \text {. }
$$

This is well defined and

$$
\tilde{\tilde{K}}(\exp 2 \pi i \theta)=\left\langle\left(\begin{array}{ccc}
\cos 2 \pi \theta & \sin 2 \pi \theta & 0 \\
-\sin 2 \pi \theta & \cos 2 \pi \theta & 0 \\
0 & 0 & 1
\end{array}\right)\right\rangle
$$

in $\operatorname{Map}^{1}\left(S^{2}, S^{2}\right) / \mathbb{Z}_{2}$ where \langle\rangle means the image under the projection $p$.

$$
\tilde{\tilde{K}}(0)=\left\langle\left(\begin{array}{lll}
1 & 0 & 0 \\
0 & 1 & 0 \\
0 & 0 & 1
\end{array}\right)\right\rangle
$$

This implies $\tilde{\tilde{K}}$ is a homotopy between following two maps

$$
\exp 2 \pi i \theta \rightarrow\left\langle\left(\begin{array}{ccc}
\cos 2 \pi \theta & \sin 2 \pi \theta & 0 \\
-\sin 2 \pi \theta & \cos 2 \pi \theta & 0 \\
0 & 0 & 1
\end{array}\right)\right\rangle
$$

and

$$
\exp 2 \pi i \theta \rightarrow\left\langle\left(\begin{array}{lll}
1 & 0 & 0 \\
0 & 1 & 0 \\
0 & 0 & 1
\end{array}\right)\right\rangle
$$

Since $p_{*}: \Pi_{1}\left(\operatorname{Map}^{1}\left(S^{2}, S^{2}\right) \rightarrow \Pi_{1}\left(\operatorname{Map}^{1}\left(S^{2}, S^{2}\right) / \mathbb{Z}_{2}\right)=\mathbb{Z}_{2}\right.$ is onto, and the $\operatorname{map} \widetilde{T}$

$$
\exp 2 \pi i \theta \rightarrow\left(\begin{array}{ccc}
\cos 2 \pi \theta & \sin 2 \pi \theta & 0 \\
-\sin 2 \pi \theta & \cos 2 \pi \theta & 0 \\
0 & 0 & 1
\end{array}\right)
$$


is the nontrivial loop in $\operatorname{Map}^{1}\left(S^{2}, S^{2}\right)$ (which we shall show later),

$$
\exp 2 \pi i \theta \rightarrow\left\langle\left(\begin{array}{ccc}
\cos 2 \pi \theta & \sin 2 \pi \theta & 0 \\
-\sin 2 \pi \theta & \cos 2 \pi \theta & 0 \\
0 & 0 & 1
\end{array}\right)\right\rangle
$$

represents the nontrivial loop, we have a contradiction. It remains to show $\widetilde{T}$ is nontrivial. Consider the nontrivial $S^{2}$-fiber bundle over $S^{2}$ with structure group $S O(3)$, i.e., the space is given as follows:

$$
\begin{aligned}
& \left(D^{2} \times S^{2}\right) \cup \cup_{T}\left(D^{2} \times S^{2}\right) \\
& \quad=\text { gluing two copies of } D^{2} \times S^{2} \text { along the boundary by } T .
\end{aligned}
$$

where $T$ is a map from $S^{1} \times S^{2}$ to itself given by

$$
(\exp 2 \pi i \theta, v) \rightarrow(\exp 2 \pi i \theta, \widetilde{T}(\exp 2 \pi i \theta)(v)) .
$$

Suppose $\widetilde{T}$ is homotopic to a constant path. Then we can get a homotopy equivalence from $\left(D^{2} \times S^{2}\right) \cup_{\mathrm{id}}\left(D^{2} \times S^{2}\right)\left(=S^{2} \times S^{2}\right)$ to $\left(D^{2} \times S^{2}\right) \cup_{T}\left(D^{2} \times S^{2}\right)$, and we have a contradiction (see $[\mathrm{S}]$ ). This completes the proof.

Remark. If we use the results in [KKR], we can give a shorter proof of the corollary above. More precisely, if $g$ can be extended, then we may construct two spaces which must be homotopy equivalent, but by the homotopy invariant in [KKR], the two spaces can not be homotopy equivalent. So we have a contradiction.

(2.3) Theorem. The diffeotopy group $\mathscr{G}$ of $S^{1} \widetilde{\times} S^{2}$ is $\mathbb{Z}_{2} \oplus \mathbb{Z}_{2}$.

Proof. Our argument is divided into 4 steps.

(1) We construct a map $\varphi$ from $\mathscr{G}$ to $\mathscr{H} / \mathbb{Z}_{2}\left(=\mathbb{Z}_{2} \oplus \mathbb{Z}_{2}\right)$.

(2) We show that the image of $\varphi$ is $\mathbb{Z}_{2}$.

(3) $\operatorname{Ker} \varphi$ is $\mathbb{Z}_{2}$.

(4) $\mathbb{Z}_{2} \longmapsto \mathscr{G} \rightarrow \mathbb{Z}_{2}$ is split.

Recall that $\mathscr{H}=\operatorname{Diff}\left(S^{1} \times S^{2}\right) / \sim$ where $\sim$ is the normal subgroup consisting of those diffeomorphisms which are diffeotopic to identity and $\mathscr{G}=$ $\operatorname{Diff}\left(S^{1} \tilde{\times} S^{2}\right) / \sim$, where $\sim$ means the same as above.

According to Gluck,

$$
\begin{aligned}
& \mathscr{H}=\mathbb{Z}_{2} \oplus \mathbb{Z}_{2} \oplus \mathbb{Z}_{2}=\langle T\rangle \oplus\langle f\rangle \oplus\langle h\rangle, \\
& S^{1} \times S^{2} \rightarrow S^{1} \times S^{2}, \\
& T:(\exp 2 \pi i \theta, v) \rightarrow\left(\exp 2 \pi i \theta,\left(\begin{array}{ccc}
\cos 2 \pi \theta & \sin 2 \pi \theta & 0 \\
-\sin 2 \pi \theta & \cos 2 \pi \theta & 0 \\
0 & 0 & 1
\end{array}\right)(v)\right) \text {, } \\
& f:(\exp 2 \pi i \theta, v) \rightarrow(\exp (-2 \pi i \theta), v), \\
& h:(\exp 2 \pi i \theta, v) \rightarrow(\exp 2 \pi i \theta,-v) \text {. }
\end{aligned}
$$


(i) First step. Define a $\mathbb{Z}_{2}$-action on $\mathscr{H}$ as follows:

$$
\begin{aligned}
& \mathbb{Z}_{2} \times \mathscr{H} \rightarrow \mathscr{H} \\
& \langle\ell\rangle \rightarrow\langle\phi \circ \ell\rangle
\end{aligned}
$$

where $\phi: S^{1} \times S^{2} \rightarrow S^{1} \times S^{2}$ is a diffeomorphism defined by

$$
(\exp 2 \pi i \theta, v) \rightarrow(-\exp 2 \pi i \theta,-v)
$$

We want to construct a map $\varphi$ from $\mathscr{G}$ to $\mathscr{H} /\langle\phi\rangle$. To do it, we have to show that, given any diffeomorphism $w$ of $S^{1} \widetilde{\times} S^{2}$, we have a lift defined up to a covering transformation, i.e.,

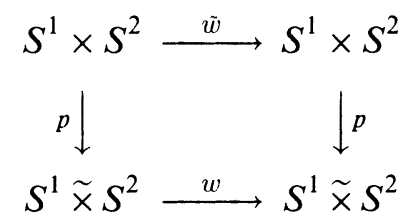

where $p: S^{1} \times S^{2} \rightarrow S^{1} \tilde{\times} S^{2}$ is the natural projection.

To show the existence of $\tilde{w}$, consider $(w \circ p)_{*} \Pi_{1}\left(S^{1} \times S^{2}\right) . S^{1} \times S^{2}$ is a double covering of $S^{1} \tilde{\times} S^{2}$, so $p_{*} \Pi_{1}\left(S^{1} \times S^{2}\right)$ is $2 \mathbb{Z} \subset \mathbb{Z}=\Pi_{1}\left(S^{1} \tilde{\times} S^{2}\right)$. Hence, any automorphism of $\Pi_{1}\left(S^{1} \widetilde{\times} S^{2}\right)$ preserves $p_{*} \Pi_{1}\left(S^{1} \times S^{2}\right)$. By the lifting lemma, there exist $\tilde{w}$ from $S^{1} \times S^{2}$ to itself such that $p \circ \tilde{w}=w \circ p$. Note that $p \circ \phi \circ \tilde{w}=w \circ p$, since $\phi$ is a regular covering transformation. Furthermore, if $w_{1}$ is isotopic to $w_{2}$ by an isotopy $H$ on $S^{1} \tilde{\times} S^{2}$, then, as in the above argument, we have $\tilde{H}: I \times S^{1} \times S^{2} \rightarrow S^{1} \times S^{2}$ such that $p \circ \tilde{H}=H \circ(\operatorname{Id} \times p)$. Now we can define a map $\varphi$ from $\mathscr{G}$ to $\mathscr{H} / \mathbb{Z}_{2}$ by $\langle\eta\rangle \rightarrow\langle\tilde{\eta}\rangle$.

Since $f \circ \phi=\phi \circ f, f$ induces a self-diffeomorphism on $S^{1} \tilde{\times} S^{2}$. Thus $\operatorname{Im} \varphi \supset \mathbb{Z}_{2}$. Note that since $h$ is isotopic to $\phi,\langle h\rangle$ is trivial in $\mathscr{H} / \mathbb{Z}_{2}$.

(ii) Second step. We know that $\operatorname{Im} \varphi \supset \mathbb{Z}_{2}$, from First step. To demonstrate $\operatorname{Im} \varphi=\mathbb{Z}_{2}$, we shall show that there exist no $T^{\prime}$ in $\mathscr{H}$ which is isotopic to $T$ and commutes with $\phi$.

Suppose that there exists such $T^{\prime}$. That means the following:

$$
\begin{gathered}
S^{1} \times S^{2} \times I \rightarrow S^{1} \times S^{2}, \\
\{(\exp 2 \pi i \theta, v), t\} \rightarrow\left(G_{t}(\exp 2 \pi i \theta, v), F_{t}(\exp 2 \pi i \theta, v)\right)
\end{gathered}
$$

where $t=0, G_{0}=\exp 2 \pi i \theta$, and

$$
\left.F_{0}(\exp 2 \pi i \theta), v\right)=\left(\begin{array}{ccc}
\cos 2 \pi \theta & \sin 2 \pi \theta & 0 \\
-\sin 2 \pi \theta & \cos 2 \pi \theta & 0 \\
0 & 0 & 1
\end{array}\right)(v)
$$


Note that $F_{0}$ is the second coordinate of $T$. For $t=1$, we have

$$
\begin{array}{ccc}
(\exp 2 \pi i \theta, v) & \longrightarrow & \left(G_{1}\left(\exp 2 \pi i \theta, F_{1}(\exp 2 \pi i \theta, v)\right)\right. \\
n & & m \\
S^{1} \times S^{2} & \longrightarrow & S^{1} \times S^{2} \\
\phi \downarrow & & \downarrow_{\phi} \\
S^{1} \times S^{2} & \stackrel{T^{\prime}}{\longrightarrow} & S^{1} \times S^{2} \\
\psi & & U \\
(-\exp 2 \pi i \theta,-v) & \longrightarrow\left(G_{1}(-\exp 2 \pi i \theta,-v), F_{1}(-\exp 2 \pi i \theta,-v)\right) .
\end{array}
$$
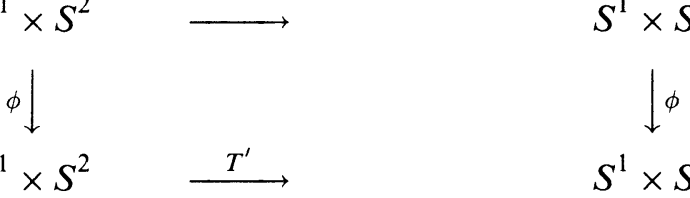

From the commutativity of the above diagram ( since $T^{\prime} \circ \phi=\phi \circ T^{\prime}$ )

$$
F_{1}(\exp 2 \pi i \theta,-v)=-F_{1}(\exp 2 \pi i \theta, v)
$$

Define $F_{t}^{\prime}$ from $S^{1}$ to $\operatorname{Map}^{1}\left(S^{1}, S^{2}\right)$ by

$$
\exp 2 \pi i \theta \rightarrow F_{t}(\exp 2 \pi i \theta, \ldots) \text {. }
$$

Note that, since $\Pi_{1}\left(\operatorname{Map}^{1}\left(S^{2}, S^{2}\right)\right)=\mathbb{Z}_{2}$ is abelian, we need not worry about choosing a base point.

Recall the $\mathbb{Z}_{2}$-action on $\operatorname{Map}^{1}\left(S^{2}, S^{2}\right)$

$$
\begin{gathered}
\mathbb{Z}_{2} \times \operatorname{Map}^{1}\left(S^{2}, S^{2}\right) \rightarrow \operatorname{Map}^{1}\left(S^{2}, S^{2}\right) \\
\lambda \rightarrow A \circ \lambda \circ A
\end{gathered}
$$

For each $t$,

$$
\begin{gathered}
{\left[F_{t}^{\prime}\right] \in \Pi_{1}\left(\operatorname{Map}^{1}\left(S^{2}, S^{2}\right)\right) \quad \text { and }} \\
{\left[p \circ F_{t}^{\prime}\right] \in \Pi_{1}\left(\operatorname{Map}^{1}\left(S^{2}, S^{2}\right) / \mathbb{Z}_{2}\right),}
\end{gathered}
$$

where [ ] means the equivalence class of loops. Then $\left[p \circ F_{1}^{\prime}\right]$ is trivial, since $p \circ F_{1}^{\prime}(-\exp 2 \pi i \theta)=p \circ F_{1}^{\prime}(\exp 2 \pi i \theta)$. Therefore $\left[p \circ F_{0}^{\prime}\right]$ is trivial, since $p \circ F_{1}^{\prime}$ is homotopic to $p \circ F_{0}^{\prime}$.

By $(2.1), \Pi_{1}\left(\operatorname{Map}^{1}\left(S^{2}, S^{2}\right) / \mathbb{Z}_{2}\right)=\mathbb{Z}_{2}$. Since $\Pi_{1}\left(\operatorname{Map}^{1}\left(S^{2}, S^{2}\right)\right)=\mathbb{Z}_{2}$ and the nontrivial element is represented by

$$
\begin{gathered}
F_{0}^{\prime}: S^{1} \rightarrow \operatorname{Map}^{1}\left(S^{2}, S^{2}\right) \\
\exp 2 \pi i \theta \rightarrow\left(\begin{array}{ccc}
\cos 2 \pi \theta & \sin 2 \pi \theta & 0 \\
-\sin 2 \pi \theta & \cos 2 \pi \theta & 0 \\
0 & 0 & 1
\end{array}\right)
\end{gathered}
$$

then, the fact that the nontrivial element in $\Pi_{1}\left(\operatorname{Map}^{1}\left(S^{2}, S^{2}\right) / \mathbb{Z}_{2}\right)$ is lifted as the nontrivial element in $\Pi_{1}\left(\operatorname{Map}^{1}\left(S^{2}, S^{2}\right)\right)$ implies a contradiction. Thus, we can conclude that $\operatorname{Im} \varphi=\mathbb{Z}_{2}$. 
(iii) Third step. Suppose $\langle q\rangle$ in $\operatorname{ker} \varphi$. Then $\tilde{q}$ is isotopic to the identity or $\phi \circ \tilde{q}$ is isotopic to the identity of $S^{1} \times S^{2}$. By a straightforward argument of Gluck (see [G, pp. 315-316] and cf. [T]), we deform $q$ so that the restriction to $\{1,-1\} \tilde{x} S^{2}$ is the identity. Since $\tilde{q}$ (or $\phi \circ \tilde{q}$ ) is isotopic to the identity, $q$ can be considered as a diffeomorphism $\bar{q}$ from $I \times S^{2}$ to $I \times S^{2}$ such that the restriction to $\{1,-1\} \times S^{2}$ of $\bar{q}$ is the identity.

By Gluck (cf. [Ha]), $\bar{q}$ is isotopic to the identity or to $\bar{d}$ while fixing the $\{1,-1\} \times S^{2}$, where $\bar{d}: I \times S^{2} \rightarrow I \times S^{2}$

$$
(t, v) \rightarrow\left(t,\left(\begin{array}{ccc}
\cos 2 \pi t, & \sin 2 \pi t & 0 \\
-\sin 2 \pi t, & \cos 2 \pi t & 0 \\
0 & 0 & 1
\end{array}\right)(v)\right) .
$$

We claim that $\bar{d}$ is $\bar{g}$, where $g$ is the self-diffeomorphism of $S^{1} \tilde{\times} S^{2}$ in (2.2). Obviously, $g$ is the identity on $\{1,-1\} \tilde{\times} S^{2} \subset S^{1} \tilde{\times} S^{2}$. Restrict $g$ to $S^{1} \widetilde{\times} S^{2}-\{1,-1\} \widetilde{\times} S^{2}$, and under the following identification,

$$
\begin{aligned}
& (\theta, v) \quad \longrightarrow \quad[(\exp 2 \pi i \theta, v)] \\
& \in \quad \in \\
& (0,1 / 2) \times S^{2} \longrightarrow S^{1} \tilde{\times} S^{2}-\{1,-1\} \tilde{\times} S^{2} \\
& (0,1 / 2) \times S^{2} \longrightarrow S^{1} \tilde{\times} S^{2}-\{1,-1\} \tilde{\times} S^{2} \\
& \epsilon \quad \epsilon \\
& \left(\theta,\left(\begin{array}{ccc}
\cos 4 \pi \theta & \sin 4 \pi \theta & 0 \\
-\sin 4 \pi \theta & \cos 4 \pi \theta & 0 \\
0 & 0 & 1
\end{array}\right)(v)\right) \\
& \rightarrow\left[\left(\exp 2 \pi i \theta,\left(\begin{array}{ccc}
\cos 4 \pi \theta & \sin 4 \pi \theta & 0 \\
-\sin 4 \pi \theta & \cos 4 \pi \theta & 0 \\
0 & 0 & 1
\end{array}\right)(v)\right)\right]
\end{aligned}
$$

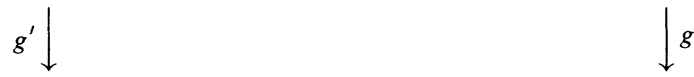

we get $g^{\prime}:(0,1 / 2) \times S^{2} \rightarrow(0,1 / 2) \times S^{2}$

$$
(\theta, v) \rightarrow\left(\theta,\left(\begin{array}{ccc}
\cos 4 \pi \theta & \sin 4 \pi \theta & 0 \\
-\sin 4 \pi \theta & \cos 4 \pi \theta & 0 \\
0 & 0 & 1
\end{array}\right)(v)\right)
$$

Identify $(0,1 / 2) \times S^{2}$ with $(0,1) \times S^{2}$ by $(\theta, v) \rightarrow(2 \theta, v)$.

Then, $g^{\prime \prime}:(0,1) \times S^{2} \rightarrow(0,1) \times S^{2}$

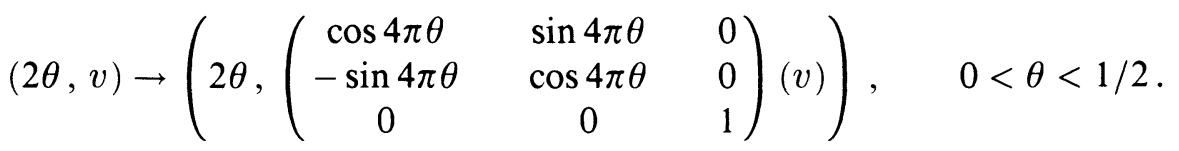


If we replace $2 \theta$ by $\theta$, we get

$$
\begin{gathered}
g^{\prime \prime}=l:(0,1) \times S^{2} \rightarrow(0,1) \times S^{2} \\
(\theta, v) \rightarrow\left(\theta,\left(\begin{array}{ccc}
\cos 2 \pi \theta & \sin 2 \pi \theta & 0 \\
-\sin 2 \pi \theta & \cos 2 \pi \theta & 0 \\
0 & 0 & 1
\end{array}\right)(v)\right)
\end{gathered}
$$

This proves the claim, i.e., $\bar{g}=\bar{d}$. Hence, $q$ is isotopic to the identity or $g ; g$ is not isotopic to the identity, otherwise $g$ could be extended to $\left(D^{2} \times S^{2}\right) / \simeq$ (see (2.2)). To show $\operatorname{Ker} \varphi=\mathbb{Z}_{2}$, it remains to show that $\langle g\rangle^{2}=$ id, i.e., $g^{2}$ is isotopic to the identity. An isotopy is constructed as follows:

$$
\begin{gathered}
S^{1} \tilde{\times} S^{2} \times I \rightarrow S^{1} \tilde{\times} S^{2} \\
\{[(\exp 2 \pi i \theta, v)], t\} \rightarrow[(\exp 2 \pi i \theta, H(\exp 4 \pi i \theta, t)(v))]
\end{gathered}
$$

where $H: S^{1} \times I \rightarrow S O(3)$ is a homotopy between the maps

$$
\begin{aligned}
& S^{1} \rightarrow S O(3) \quad \text { and } \quad S^{1} \rightarrow S O(3) \\
& \exp 2 \pi i \theta \rightarrow\left(\begin{array}{ccc}
\cos 4 \pi \theta & \sin 4 \pi \theta & 0 \\
-\sin 4 \pi \theta & \cos 4 \pi \theta & 0 \\
0 & 0 & 1
\end{array}\right), \quad \exp 2 \pi i \theta \rightarrow\left(\begin{array}{lll}
1 & 0 & 0 \\
0 & 1 & 0 \\
0 & 0 & 1
\end{array}\right)
\end{aligned}
$$

(iv) Fourth step. The splitting follows from the fact that $\langle f\rangle$ has order 2 . Now we have completed the proof.

From (2.2) and (2.3), we get the following result.

Corollary. Any self-diffeomorphism of $S^{1} \widetilde{\times} S^{2}$ homotopic to the identity is diffeotopic to the identity.

\section{REFERENCES}

[A] M. Armstrong, Lifting homotopies through fixed points, Proc. Roy. Soc. Edinburgh 92 (1982), 123-128.

[B] G. Bredon, Introduction to compact transformation groups, Academic Press, New York, 1972.

[GK] D. Gabai and W. Kazez, The classification of maps of non-orientable surface, Math. Ann. 281 (1988), 687-702.

[G] H. Gluck, Embedding of two spheres in the four sphere, Trans. Amer. Math. Soc. 104 (1962), 308-333.

[Ha] A. Hatcher, A proof of the Smale conjecture $\operatorname{Diff}\left(S^{3}\right) \simeq O(4)$, Ann. of Math. 117 (1983), 607-608.

[H] M. Hirsh, Differential topology, Springer-Verlag, 1976.

[Hu] S. T. Hu, Concerning the homotopy groups of the components of the mapping space $Y^{s^{q}}$, Nederl. Akad. Wetensch. Proc. 49 (1946), 1025-1031.

[KKR] M. Ho Kim, S. Kojima, and F. Raymond, Homotopy invariants of non-orientable 4-manifolds, Trans. Amer. Math. Soc. (to appear).

[M] J. Munkres, Topology: A first course, Prentice-Hall, 1975.

[R] J. Rubinstein, On 3-manifolds which have finite fundamental groups and contain Klein bottles, Trans. Amer. Math. Soc. 251 (1979), 129-137. 
[S] N. Steenrod, The topology of fiber bundles, Princeton Univ. Press, 1951.

[T] Y. Tao, On fixed point free involutions of $S^{1} \times S^{2}$, Osaka Math. J. 14 (1962), 145-152.

Department of Mathematics, University of Wisconsin-Parkside, Kenosha, Wisconsin 53141

Department of Mathematics, University of Michigan, Ann Arbor, Michigan 48109 (Current address of Frank Raymond)

Current address (M. Ho Kim): Department of Mathematics, Seoul National University, Seoul, Korea 